\title{
DIVISIBILITY THEORY OF ARITHMETICAL RINGS WITH ONE MINIMAL PRIME IDEAL
}

\author{
P. N. ÁNH AND M. F. SIDDOWAY \\ Version 0.0
}

\begin{abstract}
Continuing the study of divisibility theory of arithmetical rings started in [1] and [2] we show that the divisibility theory of arithmetical rings with one minimal prime ideal is axiomatizable as Bezout monoids with one minimal m-prime filter. In particular, every Bezout monoid with one minimal m-prime filter is order-isomorphic to the partially ordered monoid with respect to inverse inclusion, of principal ideals in a Bezout ring with a smallest prime ideal. Although this result can be considered as a satisfactory answer to the divisibility theory of both semi-hereditary domains and valuation rings, the general representation theory of Bezout monoids is still open.
\end{abstract}

\section{INTRODUCTION}

One of the main results in the general valuation theory developed by Krull is a dictionary between valued fields and ordered abelian groups. This dictionary was later extended by Jaffard, Kaplansky and Ohm to the larger class of Bezout domains and lattice-ordered abelian groups. This one-to-one correspondence can be considered in some sense as a local theory because all rings considered, being domains, have just one minimal prime ideal! The aim of the present note is to make this local theory complete by showing that the divisibility theory of arithmetical rings with one minimal prime ideal can be axiomatized as Bezout monoids with one minimal m-prime filter. The subclass of Bezout rings with one minimal prime ideal appears naturally in the solution of Kaplansky's question on describing commutative rings whose finitely generated modules decompose into direct sums of cyclics (see e.g. [5] .) To achieve our goal, we develop first the structure theory of Bezout monoids with one minimal m-prime filter and then, with a fairly good structural description in hand, we can complete the job by constructing, for each Bezout monoid $S$ with one minimal m-prime filter, a Bezout ring whose monoid of divisibility is order-isomorphic to $S$. Our approach is primarily influenced by the treatment of Kaplansky's problem on valuation rings presented in [7]. For the general, axiomatic theory of divisibility we refer to the fairly up-to-date presentation in Halter-Koch's book [8].

Date: Draft October 13, 2014.

2000 Mathematics Subject Classification. Primary 06F05, Secondary 13A05, 13F05, 20M14.

Key words and phrases. Bezout rings, spectrum, m-prime filter.

The first author was partially supported by the Hungarian National Foundation for Scientific Research grant no. K-101515 and VIASM (Vietnamese Institute of Advanced Study in Mathematics) for his stay in Hanoi, Vietnam during Spring 2014.

The second author was supported as the Verner Z. Reed Professor of Natural Science at Colorado College from 2007 to the present. 
A word about terminology. All structures are commutative. Rings have the identity element 1. The monoid of divisibility, or divisibility theory of a ring is simply the monoid of principal ideals under ideal multiplication partially ordered by reverse containment. A ring is called an arithmetical ring if its ideals form a distributive lattice. Every Bezout ring, i.e., a ring whose finitely generated ideals are principal, is arithmetical, but arithmetical rings needn't be Bezout rings. The set of nontrivial (i.e. different from 1 and 0 ) elements of a subset $X$ in a monoid is denoted by $X^{\star}$. Moreover, $X^{\bullet}$ will be the extension of $X$ by the new extra zero element 0 . Thus $1^{\bullet}$ is the monoid consisting of exactly two elements 1 and 0 . The positive cone of a lattice-ordered abelian group is the set of elements bigger than or equal to the identity.

\section{BASIC NOTIONS AND PRELIMINARY RESULTS}

For the benefit of the reader and for the sake of completeness we recall some easy, but basic results, notation and definitions from [1] and [2]. The development of and comments on this supporting material can be found in full detail in [1] and partly in [2].

Definition 2.1 (cf. Definition $1.1[2]$ ). A Bezout monoid $S$ (in short a B-monoid) is a commutative monoid $S$ with 0 such that the divisibility relation $a \mid b \Longleftrightarrow a S \supseteq b S$ is a partial order, called a natural partial order, inducing a distributive lattice on $S$, with a multiplication distributive on both meets and joins; and $S$ is hyper-normal, meaning that for any $a, b, d=a \wedge b \in S, a=d a_{1}$ there is $b_{1} \in S$ satisfying $a_{1} \wedge b_{1}=1, b=d b_{1}$. A monoid with 0 is called 0 -cancellative if $a x=a y \neq 0 \Rightarrow x=y$.

By Proposition 1.1 [2] the divisibility theory of a Bezout ring is a B-monoid. Moreover, by Corollary 3.4 [2] a monoid $S$ is the divisibility theory of a semi-hereditary ring if and only if it is a semi-hereditary B-monoid. Using hyper-normality, one can easily see

Corollary 2.1 (cf. Proposition $1.3[1]$ ). If 1 is a meet-irreducible element of a B-monoid $S$, that is, $s \wedge t=1 \Rightarrow s=1$ or $t=1$, then $S$ is a 0 -cancellative naturally totally ordered monoid.

This statement corresponds to a well-known result that local Bezout rings are valuation (or chained) rings. For this reason, a B-monoid is called local if 1 is meet-irreducible. Recall that a filter $F$ of a B-monoid $S$ is a subset closed under $\wedge$ such that $a \in F$ and $a \leq b \in S$ imply $b \in F$. Hence filters are also ideals in the usual sense, but the converse is in general not true. For example, the set of multiples of 3 or 5 is an ideal in the B-monoid of non-negative integers under multiplication, but it is not a filter. A filter $F$ is called an m-prime filter if $a b \in F$ implies $a \in F$ or $b \in F$. It turns out (see [1] and [2]) that m-prime filters play a decisive role in working with B-monoids. As in the case of rings, one can define the nil radical of $S$ as the intersection of all m-prime filters, and is also the intersection of all minimal m-prime filters (with respect to inclusion). As shown for commutative rings by Krull, one can see in the setting of B-monoids that the nil radical is just the set of all nilpotent elements. The general theory of m-prime filters in B-monoids can be found in [1], Sections 2 and 3.

Proposition 2.2 (cf. Theorem 2.10 [1]). If $F$ is an m-prime filter of a B-monoid $S$, then

$$
\forall x, y \in S: x \sim y \Longleftrightarrow \exists s \in S \backslash F: x \leq y s \& y \leq x s
$$


defines a congruence whose factor $S_{F}$ is a local B-monoid, and the congruence class of 0 is the set $\{x \in S \mid \exists s \notin I: s x=0\}$.

$S_{F}$ is called the localization of $S$ at an m-prime filter $F$ or at the complement $S \backslash F$ (by identifying its elements with 1 ). Using localization one can make clear the relation between annihilators and cancellation in the next assertion.

Proposition 2.3 (cf. Proposition 2.14 [1]). For arbitrary elements $v, x, y$ of a B-monoid $S$ the equality $x v=y v$ holds if and only if there is $s \in v^{\perp}=\{z \in S \mid v z=0\}$ such that $x \wedge s=y \wedge s$.

Factor lattices associated to filters are also B-monoids in view of the following result.

Proposition 2.4 (cf. Theorem 2.15 [1]). For every filter $F$ of a B-monoid $S$ a relation

$$
x \sim y \Longleftrightarrow \exists s \in F: x \wedge s=y \wedge s
$$

defines a congruence whose factor, denoted as $S / F$, is a B-monoid, called the factor B-monoid by a filter $F$ of $S$.

The key notions in our investigation are the following.

Definition 2.2. A factor set of a local B-monoid $\Sigma$ in a lattice-ordered abelian group $G$ is is the function $\mathfrak{f}: \Sigma \times \Sigma \longrightarrow G^{\bullet}$ satisfying

(FS1) $\mathfrak{f}(\alpha, \beta)=\mathfrak{f}(\beta, \alpha) \forall \alpha, \beta \in \Sigma$

(FS2) $\mathfrak{f}(\alpha, \beta \gamma) \mathfrak{f}(\beta, \gamma)=\mathfrak{f}(\alpha, \beta) \mathfrak{f}(\alpha \beta, \gamma) \quad \forall \alpha, \beta, \gamma \in \Sigma$

(FS3) $\mathfrak{f}(1, \alpha)=1, \mathfrak{f}(0, \alpha)=0 \forall \alpha \in \Sigma$

(FS4) $\mathfrak{f}(\alpha, \beta)=0$ if and only if $\alpha \beta=0$.

Two factor sets $\mathfrak{f}$ and $\mathfrak{h}$ are called associated if there is a function $\mathfrak{g}: \Sigma \longrightarrow G^{\bullet}$ with $\mathfrak{g}(1)=1, \mathfrak{g}(\alpha)=0 \Longleftrightarrow \alpha=0$ such that

$$
\mathfrak{h}(\alpha, \beta)=\frac{\mathfrak{g}(\alpha) \mathfrak{g}(\beta)}{\mathfrak{g}(\alpha \beta)} \mathfrak{f}(\alpha, \beta)
$$

holds in the case $\alpha \beta \neq 0$.

Let $T$ be the positive cone of $G$, i.e., the set of those elements in $G$ which are equal or bigger than the identity element of $G$. The (generalized) crossed product $T \underset{\mathfrak{f}}{* \Sigma}$ is

$$
S=T \underset{\mathfrak{f}}{*} \Sigma:=\{(1, t) \mid t \in T\} \dot{\cup}\left\{(\alpha, g) \mid \alpha \in \Sigma^{\star}, g \in G\right\} \dot{\cup} 0,
$$

a disjoint union of $0, T$ and copies of $G$ indexed by $\Sigma^{\star}$ endowed with the multiplication

$$
(\alpha, x) \times(\beta, y)= \begin{cases}(\alpha \beta, \mathfrak{f}(\alpha, \beta) x y), & \text { if } \alpha \beta \neq 0 ; \alpha, \beta \in \Sigma \\ 0, & \text { if } \alpha \beta=0 ; \alpha, \beta \in \Sigma\end{cases}
$$

One can easily check that $T * \underset{f}{*}$ is a B-monoid with one minimal m-prime filter where the natural partial order is exactly the lexicographic order. Two crossed products $T \underset{f}{*} \Sigma$ and $T \underset{\mathfrak{h}}{*} \Sigma$ are isomorphic if and only if $\mathfrak{f}$ and $\mathfrak{h}$ are associated. $F=S \backslash T$ after identifying $t \in T$ 
with $(1, t)$, is an m-prime (but not necessarily minimal) filter of $S$ and elements of $T$ are non-zero-divisors. $T \underset{\mathfrak{f}}{*} \Sigma$ is also a pullback in view of the next diagram

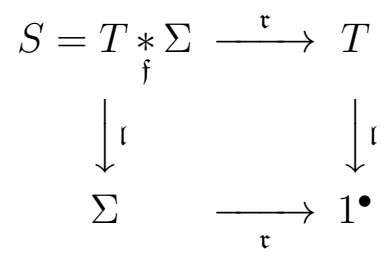

where $\mathfrak{l}$ denotes the localization maps and $\mathfrak{r}$ is the canonical map to a Rees factor.

Remark 2.3. Generalized crossed products can be formally defined as universal constructions by using factor sets in terms of arbitrary monoids $\Sigma$ and $T \subseteq U$.

\section{Structure theORY}

Throughout this section $S$ is always a B-monoid with one minimal m-prime filter $M$, with one exception in Corollary 3.2, and $T=S \backslash M$. By Proposition 2.1 the localization $\Sigma$ of $S$ at $M$ is a local B-monoid such that all non-unital elements are nilpotent. The set $Z=\{x \in S \mid \exists s \notin M: s x=0\}$ is precisely the set of elements mapped to 0 in $\Sigma$. Let $N=M \backslash Z$. Although $N$ is a distributive sublattice of $S$; it is not, in general, a subsemigroup, i.e., not closed under multiplication. Its induced partial order also fails to be natural. We shall write $a_{\sigma}$ for the image in $\Sigma$ of $a \in S$ and denote elements of $\Sigma$ by Greek letters $\alpha, \beta, \ldots$ A complete set of (pairwise different) elements of $S$ representing $\Sigma$ is called a transversal of $\Sigma$ in $S$. A transversal is normalized if $1,0 \in S$ are representatives of $1,0 \in \Sigma$, respectively. In what follows we chose an arbitrary normalized transversal $\left\{a_{\alpha} \in S \mid \alpha \in \Sigma, a_{1}=1, a_{0}=0\right\}$ and fix this transversal throughout this section. Moreover, for each $\alpha \in \Sigma$ put $S_{\alpha}=\left\{b \in S \mid b_{\sigma}=\alpha\right\}$. Thus $S_{1}=T, S_{0}=Z$ hold. Furthermore, for an element $a \in S$ we also use the notation $S_{a}=\left\{x \in S \mid x_{\sigma}=a_{\sigma}=S_{a_{\sigma}}\right\}$.

Proposition 3.1. $t<m$, or equivalently $M \subseteq$ St for all $m \in M, t \in T$.

Proof. Put $d=t \wedge m=d\left(t_{1} \wedge m_{1}\right), t=d t_{1} \in T, m=d m_{1} \in M, t_{1} \wedge m_{1}=1 . d \leq t \notin M$ and $d m_{1}=m \in M$ imply $d \notin M$ and $m_{1} \in M$ because $M$ is an m-prime filter. Thus $m_{1}^{n}=0$ for some $n \in \mathbb{N}$. Hence $t_{1}=t_{1} \wedge m_{1}^{n} \leq\left(t_{1} \wedge m_{1}\right)^{n}=1$ whence $t=d t_{1}=d<m$.

A careful analysis of the above proof leads to the following more general result.

Corollary 3.2. Let $S$ be a B-monoid with the nil radical $N$. Then for each element $m \in N$ and $t \in S$ which is not an element of the union of minimal m-prime filters of $S, t<m$, or equivalently, $N \subseteq S t$. In particular, if $t$ is a non-zero-divisor (i.e., $t^{\perp}=0$ ), then $N \subseteq S t$.

Proof. The only somewhat non-obvious claim to prove is that a non-zero-divisor $t$ is not contained in any minimal m-prime filter. Assume indirectly that there is a non-zero-divisor $t$ and a minimal m-prime filter $F$ with $t \in F$. Then the image $t_{\varphi}$ of $t$ in the localisation $S_{\varphi}$ is nilpotent contradicting the fact that $t_{\varphi}^{n} \neq 0$ for every $n \in \mathbb{N}$.

Proposition 3.3. $T$ is cancellative, that is, for any $a, x, y \in T$ an equality ax $=$ ay implies $x=y$. 
Proof. Suppose $a x=a y$ for $a, x, y \in T$. By putting $b=x \wedge y, c=x \vee y$ we have $b, c=b d \in T$ for some $d \in T$ because $M$ is m-prime. Thus $a b=a b d$, i.e., $d e=e$ for $e=a b$. Assume indirectly $x \neq y$. Then $b \neq c$ and hence $d \neq 1$. Since $e \notin M$, one obtains $e^{\perp} \subseteq M$. Therefore by Proposition 3.1, $e^{\perp} \subseteq d S$. If $F$ is a maximal proper filter containing $d S$, then in the localisation $S_{\varphi}$ of $S$ at $F$ we have $d_{\varphi} \neq 1, d_{\varphi} e_{\varphi}=e_{\gamma} \neq 0$, a contradiction to Corollary 2.1 .

Corollary 3.4. The quotient group $G$ of $T$ is lattice-ordered and $T$ is the positive cone of $G$.

Proof. By Proposition 3.3 the quotient group $G$ of $T$ exists. Write elements of $G$ in the form $s^{-1} t ; s, t \in T$. It is a tedious but routine task to check that meets and joins in $G$ obtained by putting

$$
s^{-1} t \wedge u^{-1} v=(s u)^{-1}(t u \wedge s v), s^{-1} t \vee u^{-1} v=(s u)^{-1}(t u \vee s v)
$$

are well-defined, i.e., independent of the way we write $g=s^{-1} t, g \in G$; and to verify a flock of axioms ensuring that $G$ is lattice-ordered. If $s^{-1} t \wedge 1=s^{-1}(s \wedge t)=1$, then $s=s \wedge t \leq t$ whence $t=s u$ for some $u \in T$. Consequently $s^{-1} t=u \in T$, thus $T$ is indeed, the positive cone of $G$.

Proposition 3.5. The equality $Z M=0$ holds.

Proof. Let $z \in Z, x \in M$. Then there is $s \notin M$ with $s z=0$. Since $s M=M$, there is $y \in M$ with $s y=x$. Hence $z x=z(s y)=(s z) y=0$.

Proposition 3.6. If $x \in M \backslash Z, z \in Z$, then $x<z$.

Proof. Put $x \wedge z=y$ and $x=y x_{1}, z=y z_{1}, x_{1} \wedge z_{1}=1$. $y \notin Z$ holds by $y \leq x \notin Z$. $z \in Z$ implies $t z=0$ for some $t \in T$. If $z_{1} \notin M$, then $0=t z=\left(t z_{1}\right) y$ shows $y \in Z$ because $t z_{1} \in T$, a contradiction. Thus $z_{1} \in M$ and hence $x_{1} \notin M$. By Proposition $3.1 x_{1} \leq z_{1}$ and hence $x_{1}=x_{1} \wedge z_{z}=1$ whence $x=y<z$ holds.

Proposition 3.5 implies that $Z$ can be considered as a $B$-act over both $S$ and $T^{\bullet}$ in the following sense.

Definition 3.1. Let $U$ be a B-monoid. A Bezout act over $U$, shortly a $B$-act, is a distributive lattice $A$ with the greatest element denoted also by 0 and a multiplication $U \times A \longrightarrow A$ : $(u, a) \mapsto u a \in A$ such that

$(\mathrm{BA} 0) u(v a)=(u v) a$ for all $u, v \in U ; a \in A$,

(BA1) $1 a=a, 0 a=0$ and $u 0=0$ for every $a \in A$ and $u \in U$,

(BA2) $a \leq b ; a, b \in A$ if and only if $b=s a$ for some $s \in U$,

$(\mathrm{BA} 3) \forall s, t \in U ; a, b \in A: s(a \wedge b)=s a \wedge s b, \quad s(a \vee b)=s a \vee s b,(s \wedge t) a=s a \wedge s b,(s \vee t) a=$ $s a \vee t a$,

(BA4) for any two $a, b \in A$ and $d=a \wedge b$ and $u \in U$ with $a=u d$ there is $v \in U$ that satisfies $u \wedge v=1, b=v d$.

A B-act $A$ is divisible if $u A=A$ for each non-zero-divisor $u \in U$. A is called cyclic if $A=U a$ holds for some $a \in A$, called a generator of $A$. A $U$-act map or morphism $f: A \rightarrow C$ between two acts $A$ and $C$ over $U$ is a lattice morphism satisfying $f(u a)=u f(a)$ for all $u \in U, a \in A$. In particular, a B-act morphism between cyclic B-acts over $U$ is obviously a multiplication by an appropriate element of $U$. 
Remark 3.2. Although the same symbol 0 is used for the greatest element in different structures, there is no confusion of its meaning in particular cases.

In view of Definition $3.1 Z$ is a divisible B-act over $T^{\bullet}$. In particular, $Z$ is a waist filter of $S$ in the sense that every principal filter either contains $Z$ or is a subset of $Z$. On the other hand, if $Z$ is any divisible B-act over $T^{\bullet}$ where $T$ is a positive cone of an abelian lattice-ordered group, then the disjoint union $S=T \dot{\cup} Z$ becomes a B-monoid with the smallest m-prime filter $Z$ with respect to the extended multiplication by putting $Z^{2}=0$. This B-monoid $S$ is called the trivial extension of $T$ by $Z$ (see Example 1.9 [7].) It is important to emphasize the divisibility of $Z$ which makes all elements of $T$ smaller than those of $Z$. This means that the trivial extension of a B-monoid $S$ by an $S$-act $A$ can be defined without difficulty by taking the disjoint union of $S$ and $M$ after identifying their greatest elements 0 and obviously extending the multiplication; but this new monoid is, in general, not a B-monoid. Conversely, every B-monoid with one minimal m-prime filter $M$ satisfying $M=Z=\{s \in S \mid \exists t \in T=S \backslash M: t s=0\}$, is clearly a trivial extension of $T$ by a B-act $Z=M$ over $T^{\bullet}$. Proposition 3.6 can be sharpened as

Proposition 3.7. If $x, y \in S$ such that $x_{\sigma}<y_{\sigma}$, then $x<y$.

Proof. Put $u=x \wedge y=u\left(x_{1} \wedge y_{1}\right), x=u x_{1}, y=u y_{1}, x_{1} \wedge y_{1}=1$. Then one of $x_{1}, y_{1}$ is in $M$ otherwise we have $y_{\sigma}=x_{\sigma} \vee y_{\sigma}=(x \vee y)_{\sigma}=\left(u x_{1} y_{1}\right)_{\sigma}=u_{\sigma}=x_{\sigma}$, a contradiction. On the other hand, one of $x_{1}, y_{1}$ is not in $M$ by $x_{1} \wedge y_{1}=1$. Thus, either $x_{1}=1$ or $y_{1}=1$. The latter is impossible because $y=u y_{1}=u$ would imply $y_{\sigma}=u_{\sigma}=x_{\sigma}$, a contradiction. Thus $x_{1}=1$ and hence $x<y$.

The following weak cancellation property is important in the structural study of B-monoids with one minimal m-prime filter.

Proposition 3.8. Let $x \in M \backslash Z$, i.e., $x^{\perp} \subseteq M$. For any $s, t \in T$ there is precisely one $y \in M$ satisfying $t x=$ sy. Moreover, an equality $t x=s x$ for $t, s \in T$ implies $t=s$. In particular, $x y=y \notin Z$ implies $x=1$.

Proof. Assume indirectly $t x=s y_{1}=s y_{2}$ and $y_{1} \neq y_{2}$. If $v=y_{1} \vee y_{2}>u=y_{1} \wedge y_{2} \in M$, then $v=u z$ with $1 \neq z$. Furthermore, we have $s u=s v=(s u) z=t x \neq 0$. Therefore $(s u)^{\perp}=(t x)^{\perp} \subseteq M$. The filter generated by $z \wedge M$ is proper because either $z \in M$ or $M \subseteq S z$. Hence this filter can be extended to an m-prime filter $F$. In the localization $S_{F}$ of $S$ at $F$ we have $(s u)_{\varphi} z_{\varphi}=(s u)_{\varphi}=(t x)_{\varphi} \neq 0$ with $z_{\varphi} \neq 1$, a contradiction. For the second assertion, Proposition 2.3 implies $t \wedge y=s \wedge y$ for some $y \in x^{\perp}$. Since $y<s$ and $y<t$ by Proposition 3.7, one has $t=s$.

For every element $s \in T$ we have $M \subseteq s S$. Hence for any element $x \in M$ there is an element $y \in M$ with $x=s y$. Therefore, one can define the action of $G$ on the distributive lattice $N=M \backslash Z$ by putting $g x=y$ for every $g=s^{-1} t \in G$ and $x \in N$ if $t x=s y$. This definition is well-defined, i.e., $y$ is independent of the representation of $g$ in the form $g=s^{-1} t$. For, if $g=s^{-1} t=u^{-1} v$ with $s, t, u, v \in T$, then $u t=s v$ holds and the equalities $t x=s y, v x=u z$ imply $y, z \in N$ and $s v y=t v x=t u z=s v z$ showing $y=z$ by Proposition 3.7. It is obvious that for each $a \in N$ the orbit of $a$ is just $S_{a}$. Moreover, for any $y=g x=s^{-1} t x, a \in N$ we have $t a=s b$ for $b=g a \in N$. By putting $d=s \wedge t=d\left(s_{1} \wedge t_{1}\right), s=d s_{1}, t=d t_{1}, s_{1} \wedge t_{1}=1$ we 
obtain $g=s^{-1} t=\left(d s_{1}\right)^{-1}\left(d t_{1}\right)=s_{1}^{-1} t_{1}$. Therefore one can assume without loss of generality that $g=s^{-1} t$ with $s \wedge t=1$. Consequently we have

$$
\begin{aligned}
& (g x) a=y a=y a(s \wedge t)=y a s \wedge y a t=t x a \wedge y a t= \\
& =s b x \wedge s b y=s b x \wedge b t x=b x(s \wedge t)=x b=x(a g) .
\end{aligned}
$$

Note that an equality $(g x) a=g(x a)$ with $a, x \in N$ does in general, not hold. The reason is that although the left hand side is always meaningful, the right hand side is not defined for the case when $0 \neq x a \in Z$. In fact, it is impossible to define an action of $G$ on $Z$ in the case $Z \neq 0$. For example, there are B-monoids $S$ with $Z \neq 0$ and some elements $a, x \in N, g \in G$ such that $0 \neq(g a) x=a(g x) \in Z$, but $a x=0$. However, the equality $(g x) a=x(a g)$ always holds, as we have already seen above. Furthermore, each $x \in S_{a}$ defines a bijective map $\jmath_{x}: S_{a} \longrightarrow G$, sending $y=g x \in S_{\alpha}$ (i.e., $y$ satisfies $t x=s y$ if $\left.g=s^{-1} t\right)$ to $g \in G$. We summarize these results in the following theorem.

Theorem 3.9. For a B-monoid $S$ with one minimal m-prime filter $M$ put $N=M \backslash Z$. The quotient group $G$ of $T=S \backslash M$ acts on $N$ by putting ga $=b$ for $a \in N, g=s^{-1} t$ if $t a=s b$. The orbit of $a \in N$ is just $S_{a}$. Each $x \in S_{a}$ induces the lattice isomorphism

$$
\jmath_{x}: S_{a} \longrightarrow G: y=g x \in S_{a} \mapsto \jmath_{x}(y)=g .
$$

Moreover, for every $g \in G ; a, b \in N$ the action of $G$ on $N$ satisfies the equality

$$
(g a) b=a(g b) \text {. }
$$

If $a b \notin Z$, then $(g a) b=g(a b)=a(b g)$.

The already fixed transversal $\left\{a_{\alpha}, \alpha \in \Sigma\right\}$ immediately defines a factor set $\mathfrak{f}: \Sigma \times \Sigma \longrightarrow$ $G \dot{\cup} 0$ by putting $\mathfrak{f}(\alpha, \beta)=0$ if $\alpha \beta=0 \in \Sigma$, or equivalently $a_{\alpha} a_{\beta} \in Z$ and requiring $a_{\alpha} a_{\beta}=\mathfrak{f}(\alpha, \beta) a_{\alpha \beta}$ for the case $0 \neq \alpha \beta \in \Sigma$, or equivalently $a_{\alpha} a_{\beta} \notin Z$. If $\mathfrak{h}$ is a factor set induced by another normalized transversal $\left\{b_{\alpha} \mid \alpha \in \Sigma\right\}$, then for each element $\alpha \in \Sigma^{\star}$ there is a uniquely determined element $\mathfrak{g}(\alpha) \in G$ such that $b_{\alpha}=\mathfrak{g}(\alpha) a_{\alpha}$. If in addition we put $\mathfrak{g}(1)=1, \mathfrak{g}(0)=0$ we obtain the function $\mathfrak{g}: \Sigma \longrightarrow G^{\bullet}$. For $\alpha \beta \neq 0 \in \Sigma$ the equalities

imply

$$
\begin{aligned}
\mathfrak{h}(\alpha, \beta) \mathfrak{g}(\alpha \beta) a_{\alpha \beta} & =\mathfrak{h}(\alpha, \beta) b_{\alpha \beta}=b_{\alpha} b_{\beta}=g(\alpha) g(\beta) a_{\alpha} a_{\beta}= \\
& =g(\alpha) g(\beta) \mathfrak{f}(\alpha, \beta) a_{\alpha \beta},
\end{aligned}
$$

$$
\mathfrak{h}(\alpha, \beta)=\frac{\mathfrak{g}(\alpha) \mathfrak{g}(\beta)}{\mathfrak{g}(\alpha \beta)} \mathfrak{f}(\alpha, \beta),
$$

hence $\mathfrak{f}$ and $\mathfrak{h}$ are associated. Therefore we have proved, in every detail, the next theorem.

Theorem 3.10. The Rees factor $R$ of $S$ by $Z$ is isomorphic to the generalized crossed product $T * \Sigma$ of $T$ by $\Sigma$ with the factor set $\mathfrak{f}$ induced by a normalized transversal of $\Sigma$ in $S$. Conversely, if $P$ is the positive cone of a lattice-ordered group $G, \Delta$ is a local B-monoid whose non-unital elements are nilpotent, and $\mathfrak{f}$ is a factor set of $\Delta$ in $G^{\bullet}$, then the crossed product $C=P * \Delta$ is a B-monoid having one minimal m-prime filter $M_{C}=C \backslash P$, the localisation $C_{\delta}$ of $C$ at $M_{C}$ satisfies $C_{\delta} \cong \Delta$, and every element of $a \in P \subseteq C$ is a non-zero-divisor.

Remark 3.3. The Rees factor of $S$ by $Z$ is exactly the factor of $S$ by the filter $Z$ in view of Propositions 2.4 and 3.6. 
The following result provides some more information about the structure of $Z$.

Proposition 3.11. Let $S$ be a B-monoid with the smallest m-prime ideal $M$. Let $Z=\{x \in$ $S \mid \exists s \notin M: s x=0\}, T=S \backslash M$. If there is $u \in M$ such that $s u \neq 0$ for all $s \in T$, or equivalently $\Sigma \neq 1^{\bullet}$, then $Z$ is a factor of the quotient group $G$ of $T$ by an appropriate filter $F$ of $G: g, h \in G$ map to the same element of $Z$ iff there is an element $k \in F$ satisfying $g \wedge k=h \wedge k$.

Proof. The case $Z=0$ is obvious. Thus without loss of generality one can assume that $Z \neq 0$. By the assumption as well as by Propositions 3.5, 3.6 there are two elements $a, b \in M \backslash Z$ with $0 \neq a b \in Z$. In view of Propositions 3.5 and 3.7 we have $a c=0$ if $c_{\sigma}>b_{\sigma}$, and $a S_{b}=Z$ from which the assertion follows immediately by Proposition 2.3.

The above statement suggests the divison of B-monoids with one minimal m-prime filter into three types.

Definition 3.4. Let $S$ be a B-monoid with one minimal m-prime filter $M$ and consider $Z=\{x \in S \mid \exists s \notin M: s x=0\} \subseteq M . S$ is said to be of

(1) type $I$ if $Z=0$

(2) type $I I$ if $0 \neq Z=M$, and

(3) type $I I I$ if $0 \neq Z \neq M$.

By definition $S$ is of type II if every element of the least minimal m-prime filter $M \neq 0$ is annihilated by some element in $T=S \backslash M$. This is equivalent to saying that $\Sigma$ is just the trivial B-monoid $1^{\bullet}$. By Proposition 3.5, $M^{2}=M Z=0$. Therefore $M$ can be considered as a divisible B-act over $T^{\bullet}$ and $S$ is a trivial extension of $T$ by $M=Z$. Therefore every B-monoid with one minimal m-prime ideal of type II can be obtained in this manner. The structure of B-monoids with one minimal m-prime filter of type II can be described more precisely with the help of direct limits as follows. First note that the set $I=M^{\star}=Z^{\star}$ of nonzero elements of $M=Z$ can be identified with the set of all nonzero cyclic acts $S a=T a, 0 \neq a \in M$ and the partial order given by set-theoretic inclusion on $I$ is just the reverse order of the original ordering on $I . \quad I$ is indeed a down-directed set and for any $a \leq b$ in $I$ there is $t_{b}^{a} \in T$ with $a t_{b}^{a}=b$. Therefore by assigning to each $a \in I$ the factor B-act $T_{a}$ of $T$ via $t \sim s \Longleftrightarrow \exists u \in a^{\perp} \cap T: t \wedge u=s \wedge u$ and the B-act map $\tau_{b}^{a}: T_{b} \rightarrow T_{a}$ given by multiplication with $t_{b}^{a}$ for any pair $a \leq b, b=b t_{b}^{a}, t_{b}^{a} \in T$ one obtains a direct system of B-acts over $T^{\bullet}$ whose limit is canonically isomorphic to $M$. In fact, the element $a \in I$ is identified with the equivalence class of the image of $1 \in T$ in $T_{a}$. We have therefore the following description of type II.

Proposition 3.12. If $S$ is a B-monoid with one minimal m-prime filter $M$ of type II, then there is a direct system of nonzero cyclic $B$-acts $T_{a}, 0 \neq a \in M$ over $T^{\bullet}$ together with the injective $B$-act maps $\tau_{b}^{a}: T_{b} \rightarrow T_{a}$ given by multiplication with elements $t_{b}^{a} \in T$ such that $b=a t_{b}^{a}$ for any pair of elements $a \leq b=a t_{b}^{a}$ and $S$ is isomorphic to the trivial extension $T \cup \lim T_{a}$ sending $M$ to $\lim _{a} T_{a}$. In particular, every $B$-monoid with one minimal m-prime filter of type II can be obtained in this manner.

For type II we do not know if $Z$ is also a factor (as a B-act over $T$ ) of the quotient group $G$ of $T$. For type III we need the following preparation. 
Proposition 3.13. Let $S$ be of type III, that is, $0 \neq Z=\{x \in S \mid \exists s \notin M: s x=0\} \neq M$. Then for each $\alpha \in \Sigma^{\star}$ there is exactly one $\alpha_{i} \in \Sigma^{\star}$ such that for every $a \in S_{\alpha}$ there is $b \in S_{\alpha_{i}}$ with $0 \neq a b \in Z$. In particular, the assignment $\imath: \alpha \in \Sigma^{\star} \mapsto \alpha_{i} \in \Sigma^{\star}$ is a bijection of $\Sigma^{\star}$ satisfying $\imath^{2}=\imath$.

Proof. Let $a \in S_{\alpha}$ be an arbitrary element. Then $a \in N=M \backslash Z$ holds. By Proposition 3.6 $a<z$ for any non-zero element $z \in Z$, hence there is $b \in S$ with $a b=z \neq 0$. By Proposition $3.5, b \notin Z$. On the other hand, if $b \notin M$, then $b_{\sigma}=1$ and thus $0=(a b)_{\sigma}=a_{\sigma} b_{\sigma}=a_{\sigma} \neq 0$, a contradiction. Consequently we have $b \in M$, hence $\alpha_{i}=b_{\sigma} \in \Sigma^{\star}$. If $\alpha_{i}<\beta$ and $c \in S_{\beta}$ is an arbitrary element, then by Proposition 3.7 there is $x \in M$ with $c=b x$, from which $a c=a b x=0$ holds by Proposition 3.5. Thus $a c=0$ for all $a \in S_{\alpha}$ and $c \in S_{\beta}$ if $\alpha_{i}<\beta$. If $\beta<\alpha_{i}$ and $c \in S_{\beta}$, then by the same argument as above one obtains $a c \notin Z$, otherwise $a b=0$ results which is impossible by the choice of $b$. Thus we have shown the uniqueness of $\alpha_{i}$ which completes the proof if we interchange the roles of $\alpha$ and $\alpha_{i}$.

As an immediate consequence of the above proof we obtain

Corollary 3.14. Let $\Sigma=\Sigma \dot{U} \infty$ be the commutative extension of $\Sigma$ by the greatest nonzero element $\infty$ subject to $\alpha \alpha_{i}=\infty$ and $\alpha \infty=0=\alpha \beta$ for all $\alpha \in \Sigma, \beta \in \Sigma, \alpha_{i}<\beta$. Then $\Sigma$ is a local B-monoid and its Rees factor by the filter $\{\infty, 0\}$ is $\Sigma$.

For the sake of simplicity we will write $\jmath_{\alpha}$ instead of $\jmath_{a_{\alpha}}$ for maps $\jmath_{x}$ defined in Theorem 3.9. Note that $\left\{a_{\alpha}, \alpha \in \Sigma\right\}$ is the transversal of $\Sigma$ in $S$ already under consideration throughout this section. For each $\alpha \in \Sigma^{\star}$ we define, by using the bijection $\imath: \alpha \mapsto \alpha_{i}$ established in Proposition 3.13, the $G$-pairing

$$
\mathfrak{m}_{\alpha}: S_{\alpha} \times S_{\alpha_{i}} \longrightarrow G:(a, b) \mapsto \mathfrak{m}_{\alpha}(a, b)=\jmath_{\alpha}(a) \jmath_{\alpha_{i}}(b) \in G
$$

and the $T$-act epimorphism

$$
\mathfrak{c}_{\alpha}: G \longrightarrow Z: g \in G \mapsto a_{\alpha}\left(g a_{\alpha_{i}}\right) \in Z .
$$

In view of Propositions 3.13 and 2.3 one can see immediately that factors of $G$ by the congruences $g \sim_{\alpha} h \Longleftrightarrow \exists k \in C_{\alpha}: g \wedge k=h \wedge k$ are isomorphic to $Z$ as B-acts over $T^{\bullet}$ where $C_{\alpha}=\left\{g \in G \mid \mathfrak{c}_{\alpha}(g)=0\right\}$. By Theorem 3.9, for any $\alpha \in \Sigma^{\star}$ and arbitrary elements $a \in S_{\alpha}, b \in S_{\alpha_{i}}$ it follows that

$$
\mathfrak{c}_{\alpha}\left(\mathfrak{m}_{\alpha}(a, b)\right)=\mathfrak{c}_{\alpha}\left(\jmath_{\alpha}(a) \jmath_{\alpha_{i}}(b)\right)=a_{\alpha}\left[\jmath_{\alpha}(a)\left(\jmath_{\alpha_{i}}(b) a_{\alpha_{i}}\right)\right]=a_{\alpha}\left(\jmath_{\alpha}(a) b\right)=\left(a_{\alpha} \jmath_{\alpha}(a)\right) b=a b \in Z .
$$

If $\alpha<\beta ; \alpha, \beta \in \Sigma^{\star}$, then $\beta=\alpha \gamma$ for some $\gamma \in \Sigma^{\star}$ and so $\alpha_{i}=\gamma \beta_{i}$ holds. Hence

$$
a_{\alpha} a_{\gamma}=\mathfrak{f}(\alpha, \gamma) a_{\beta} \quad \& \quad a_{\beta_{i}} a_{\gamma}=\mathfrak{f}\left(\beta_{i}, \gamma\right) a_{\alpha_{i}} .
$$

To aid the exposition, we write

$$
h_{\beta}=\mathfrak{f}(\alpha, \gamma)^{-1} \mathfrak{f}\left(\beta_{i}, \gamma\right) .
$$

One has for each $g \in G$, the following equalities

$$
\begin{gathered}
\mathfrak{c}_{\beta}(g)=a_{\beta}\left(g a_{\beta_{i}}\right)=\left(\mathfrak{f}(\alpha, \gamma)^{-1} a_{\alpha} a_{\gamma}\right)\left(g a_{\beta_{i}}\right)=a_{\alpha}\left(\mathfrak{f}(\alpha, \gamma)^{-1} g a_{\gamma} a_{\beta_{i}}\right)=a_{\alpha}\left(\mathfrak{f}(\alpha, \gamma)^{-1} \mathfrak{f}\left(\beta_{i}, \gamma\right) g a_{\alpha_{i}}\right)= \\
=a_{\alpha}\left(h_{\beta} g a_{\alpha_{i}}\right)=\mathfrak{c}_{\alpha}\left(h_{\beta} g\right)
\end{gathered}
$$


whence $\mathfrak{c}_{\beta}=\mathfrak{c}_{\alpha} h_{\beta}$ and $\mathfrak{c}_{\alpha}=\mathfrak{c}_{\beta} \mathfrak{f}(\alpha, \gamma) \mathfrak{f}\left(\beta_{i}, \gamma\right)^{-1}$ holds for all $\alpha<\beta ; \alpha, \beta \in \Sigma^{\star}$. Therefore, for arbitary elements $\alpha, \beta \in \Sigma^{\star}$ one has

$$
\mathfrak{c}_{\beta}=\mathfrak{c}_{\alpha} h_{\beta} \text { where } h_{\beta}=\left\{\begin{array}{l}
\mathfrak{f}(\alpha, \gamma)^{-1} \mathfrak{f}\left(\beta_{i}, \gamma\right) \text { if } \alpha<\beta=\alpha \gamma \\
\mathfrak{f}\left(\alpha_{i}, \gamma\right)^{-1} \mathfrak{f}(\beta, \gamma) \text { if } \beta<\alpha=\beta \gamma
\end{array}\right.
$$

The above considerations allow us to define a new B-monoid $\mathfrak{S}$ with one minimal mprime filter of type I having the epimorphic image $S$ as follows. Consider the disjoint union $\mathfrak{S}=S \dot{\cup} S_{\infty}$ where $S_{\infty}=G$, and define $S_{\beta} S_{\infty}=0$ for all $\beta \in \Sigma^{\star}$. For $s \in T=S_{1}$ and $g \in S_{\infty}$ define their product as the usual group product $s g \in S_{\infty}$. We fix now one (arbitrarily chosen) element $\alpha \in \Sigma^{\star}$ and define for each $\beta \in \Sigma^{\star}$ and arbitrary elements $x \in S_{\beta}, y \in S_{\beta_{i}}$ the (commutative) product $x y=h_{\beta} \jmath_{\beta}(x) \jmath_{\beta_{i}}(y)$ according to the equality $(*)$. Note that $S_{\beta} S_{\gamma}=0$ for all $\gamma>\beta_{i}$ and $x y \in S \backslash Z$ for all $x \in S_{\beta}, y \in S_{\gamma}$ with $\gamma<\beta_{i}$. Furthermore, $T$ is the set of all non-zero-divisors of $\mathfrak{S}$ and the localization of $\mathfrak{S}$ at $T$ is $\Sigma=\Sigma \dot{U} \infty$ with the greatest non-zero element $\infty$. Moreover, for this fixed element $\alpha \in \Sigma^{\star}$, the factor of $\mathfrak{S}$ by the filter $C_{\alpha}=\left\{g \in G=S_{\infty} \mid \mathfrak{c}_{\alpha}(g)=0\right\}$ is $S$. Note that this factor is in general, not a Rees factor unless $G$ is a totally ordered abelian group. Thus we have shown that B-monoids with one minimal m-prime filter of type III are factors of particular B-monoids with one minimal mprime filter of type I. Therefore one can describe a structure of B-monoids with one minimal m-prime filter as follows.

Theorem 3.15. Let $S$ be a B-monoid with one minimal m-prime filter $M, T=S \backslash M$ and $Z=\{x \in S \mid \exists s \notin M: s x=0\}$. Let $\Sigma$ be a localization of $S$ at $M$. Then $S$ belongs exactly to one of the following three cases.

(1) $Z=0$. Then $S$ is a crossed product of $T$ with $\Sigma$.

(2) $Z=M$. Then $S \cong T \ltimes \lim _{\longrightarrow}\left\{T_{a}, t_{b}^{a}\right\}$ where the latter term is a divisible direct limit of factors $T_{a}$ of $T$ by $x \cong y \Longleftrightarrow \exists z \in L_{a}=a^{\perp} \cap T: x \wedge z=y \wedge z$ given by $a$ down-directed set $I=M^{\star}=\{0 \neq a \in M\}$ with elements $t_{b}^{a} \in T$ such that $b=a t_{b}^{a}$ for all $a<b$ in $I$.

(3) $0 \neq Z \neq M$. Then $\Sigma$ is the factor of the local B-monoid $\Sigma$ extended by the greatest nonzero element $\infty$. Moreover $S$ is the factor of the B-monoid $\mathfrak{S}$ with one minimal m-prime filter of type $I$ by a filter contained in $S_{\infty}$.

\section{A REPRESENTATION THEOREM}

The aim of this section is to prove the following theorem, main result of this note.

Theorem 4.1. The divisibility theory of an arithmetical ring with one minimal prime ideal is a Bezout monoid with one minimal m-prime filter. Conversely, every Bezout monoid with one minimal m-prime filter is order-isomorphic to the divisibility theory of an appropriate Bezout ring.

Proof. Let $R$ be an arithmetical ring with one minimal prime ideal $I$ and $S(R)$ its monoid of divisibility. It is shown in [1] Proposition 1.1 that $S(R)$ is a B-monoid, and hence $S(R)$ has only one minimal m-prime filter $F$ consisting of principal ideals contained in $I$, as is easy to check. For the sufficiency, let $S$ be an arbitrary B-monoid with one minimal m-prime filter $M$ and $K$ an arbitrary field. As a first step we need 
Lemma 4.2 (cf. Gauss' Lemma on primitive polynomials). Let $R$ be the 0 -contracted monoid algebra of $S$ over $K$, i.e., $R$ consists of all linear combinations of nonzero elements of $S$ with non-zero coefficients from $K$ where the zero elements of both $S$ and $K$ are identified. Then the set of primitive elements of $R$ is multiplicatively closed.

Remark 4.1. $\sum_{i=1}^{i=n} k_{i} s_{i} \in R\left(0 \neq k_{i} \in K^{\star}, i=1, \ldots, n\right)$ is called primitive if $\wedge_{i=1}^{n} s_{i}=1$. Primitive elements are in general not regular, that is, they are not necessarily non-zerodivisors. For example, if $s t=t \neq 0 ; t, s \in S$, then $1-s$ is obviously primitive and a zerodivisor by $t(1-s)=0$. Let $\mathfrak{P}$ be the monoid of principal ideals of the ring $\mathbb{Z} \ltimes \mathbb{Q} / \mathbb{Z}$, i.e., $\mathfrak{P}$ is generated by $s_{p}, t_{p^{-n}} ; n \in \mathbb{N}, p \in \mathbb{P}=\{$ prime numbers $\}$ subject to $s_{p} t_{p}=t_{p} t_{q}=0 \forall p, q \in \mathbb{P}$ and $s_{p} t_{p^{-n}}=t_{p^{n-1}}, s_{p} t_{q}=t_{q} \forall p \neq q \in \mathbb{P} ; n>1$. $\mathfrak{P}$ is a B-monoid with one minimal m-prime filter $M$ generated by all $t_{p^{-n}}$ and every element of $\mathfrak{P}$ different from 0 and 1 is a zero-divisor! Thus in the 0 -contracted monoid algebra $K \mathfrak{P}$ there are many primitive elements which are zero-divisors! For more on the important role of Gauss' Lemma in valuation theory, we refer to the forthcoming paper [3]

Proof. Since $M \in s S$ for all $s \notin M$, an element $r=\sum_{i=1}^{i=n} k_{i} s_{i}$ is primitive if and only if the image of $r$ in the monoid algebra $K T, T=S \backslash M$ is also primitive. By this observation, the assertion follows immediately from the well-known corresponding statement for lattice ordered groups, see for example [9], Theorem 8.1. For another short and conceptual proof of this important result, see [3].

We are now in position to verify the main result. We have to provide a representation of $S$ as a monoid of divisibility of a Bezout ring in three cases according to the type of $S$ in view of Theorem 3.15 .

Case 1: $S$ is of type I. Let $R$ be a 0 -contracted monoid algebra of $S$ over $K$. We claim that primitive elements of $R$ are non-zero-divisors. Consider an arbitrary primitive element $a=\sum_{i=1}^{i=n} k_{i} s_{i},\left(k_{i} \in K^{\star}, 1 \leq n \in \mathbb{N}\right)$. Since products of primitive elements are again primitive by Lemma 4.2 and each element of $R$ can be written as a product of a primitive element with a monoid element, it is enough to see $a s \neq 0$ for any $s \in S^{\star}$. If $s \in T$, then $s x \neq s y$ for any two different elements $x, y$ of $S$ because $S$ is of type I. Thus as $\neq 0$ for all $s \notin M$. Assume $0 \neq s \in M$. Write $a=a_{1}+a_{2}$ where $a_{1}$ and $a_{2}$ are linear combinations of the $k_{i} s_{i}$ with $s_{i} \notin M$ and $s_{i} \in M$, respectively. Since $a$ is primitive, $a_{1} \neq 0$ whence $s a_{1} \neq 0$ in view of Proposition 3.7. Observing that $\left(s s_{i}\right)_{\alpha}=s_{\alpha}$ for $s_{i} \notin M$ and $s s_{i}>s$ for $s_{i} \in M$, one gets $a s \neq 0$. Thus every primitive element of $R$ is a non-zero-divisor. Let $A$ be the localization of $R$ at the set of all primitive elements. It is clear that the monoid of principal ideals of $A$ is isomorphic to $S$ and $S$ can be considered as a submonoid with respect to the multiplication of $A$.

Case 2: $S$ is of type III. By Theorem $3.15 S$ is a factor of the B-monoid $\mathfrak{S}$ with one minimal m-prime filter of type I by a filter $C$ contained in $S_{\infty}$. According to Case 1 there is a Bezout ring $R$ containing a submonoid $\mathfrak{S}$ such that principal ideals of $R$ are exactly those 
generated by elements of $\mathfrak{S}$. The factor ring $A$ of $R$ by the ideal generated by elements of $C$ obviously has divisibility theory isomorphic to $S$.

Case 3: $S$ is of type II. We will use the notation and the description of $S$ given in Theorem 3.15 (2). Let $T=S \backslash M$ and $B$ be the localisation of the monoid algebra $K T$ at the set of all primitive elements. Then $B$ is a Bezout domain whose divisibility theory is $T^{\bullet}$. For each $0 \neq a \in M$ let $B_{a}$ be the cyclic factor of $B$ by the ideal generated by $t \in a^{\perp}$. Multiplication by $t_{b}^{a} \in T \subseteq K T$ induces an injective module homomorphism $t_{b}^{a}: B_{b} \longrightarrow B_{a}$ for any two non-zero elements $a \leq b=t_{b}^{a} b$ of $M$. Let $C$ be the direct limit of the $B$-modules ${ }_{B} B_{a}$. Then it is routine to check that ${ }_{B} C$ is divisible, and the lattice of $B$-submodules of $C$ is isomorphic to $M=Z$. Let $Q$ be the trivial extension of $B$ by $C$, then the divisibility theory of $Q$ is just $S$.

The above proof suggests a broader and sharper version of Kaplansky's original question on valuation rings as follows.

Kaplansky's problem. Describe all factors of Bezout domains or more generally, all factors of semi-hereditary Bezout rings as well as of semi-hereditary Bezout monoids. In particular, one can search for the description of all factors having one minimal prime ideal (m-prime filter). For details on the original problem of Kaplansky, we refer to the book [7].

\section{REFERENCES}

1. P. N. Ánh, L. Márki, P. Vámos, Divisibility theory in commutative rings: Bezout monoidss, Trans. Amer. Math. Soc. 364(2012), 3967 - 3992.

2. P. N. Ánh, M. Siddoway, Divisibility theory of semi-hereditary rings, Proc. Amer. Math. Soc. 138 (2010), $4231-4242$

3. P. N. Ánh, M. Siddoway, Gauss' Lemma and valuation theory, submitted.

4. G. Birkhoff, Lattice theory, AMS, Colloq. Publ. 25, 3rd edition, 1967.

5. W. Brandal, Commutative rings whose finitely generated modules decompose, LNM 723, Springer, 1979.

6. A. H. Clifford, Naturally totally ordered commutative semigroups, Amer. J. Math. 76 (1954), $631-646$

7. L. Fuchs and L. Salce, Modules over non-Noetherian domains, Mathematical Surveys and Monographs 84, AMS, 2001.

8. F. Halter-Koch, Ideal Systems. An Introduction to Multiplicative Ideal Theory, Pure and Applied Mathematics, 211, Marcel Dekker, New York, 1998.

9. J. Močkoř, Groups of divisibility, D. Reidel 1983.

10. T. S. Shores, On generalized valuation rings, Michigan Math. J. 21 (1974), $405-409$.

11. W. Stephenson, Modules whose lattice of submodules is distributive, Proc. London Math. Soc. 27 (1974), 291-310.

Rényi Institute of Mathematics, Hungarian Academy of Sciences, 1364 Budapest, Pf. 127 HUNGARY

E-mail address: anh.pham.ngoc@renyi.mta.hu

Department of Mathematics and Computer Science, Colorado College, Colorado Springs, CO 80903.

E-mail address: msiddoway@coloradocollege.edu 